Draft version August 8, 2021

Typeset using LATEX preprint style in AASTeX61

\title{
ANALYSIS OF LOW EXCITATION HDO TRANSITIONS TOWARD THE HIGH-MASS STAR-FORMING REGIONS G34.26+0.15, W51E $\mathrm{E}_{1} / \mathrm{E}_{2}$, AND W49N
}

\begin{abstract}
MAGDA KULCZAK-JASTRZȨBSKA ${ }^{1}$
${ }^{1}$ Astronomical Observatory of the Jagiellonian University, 30-244 Orla 171, Kraków, Poland; kulczak@oa.uj.edu.pl

(Received)

ABSTRACT

We present observations of the ground state $1_{0,1}-0_{0,0}$ rotational transition of HDO at 464.925 $\mathrm{GHz}$ and the $1_{1,0}-1_{0,1}$ transition at $509.292 \mathrm{GHz}$ towards the three high-mass star forming regions: G34.26+0.15, W49N and $\mathrm{W} 51 \mathrm{e}_{1} / \mathrm{e}_{2}$, carried out with the Caltech Submillimeter Observatory. The latter transition is observed for the first time from the ground. The spectra are modeled, together with observations of higher-energy HDO transitions, as well as submillimeter dust continuum fluxes from the literature, using a spherically symmetric radiative transfer model to derive the radial distribution of the HDO abundance in the target sources. The abundance profile is divided into an inner hot core region, with kinetic temperatures higher than $100 \mathrm{~K}$ and a cold outer envelope with lower kinetic temperatures. The derived HDO abundance with respect to $\mathrm{H}_{2}$ is $(0.3-3.7) \times 10^{-8}$ in the hot inner region $(T>100 \mathrm{~K})$ and $(7.0-10.0) \times 10^{-11}$ in the cold outer envelope. We also used two $\mathrm{H}_{2}^{18} \mathrm{O}$ fundamental transitions to constrain the $\mathrm{H}_{2} \mathrm{O}$ abundances in the outer envelopes. The $\mathrm{HDO} / \mathrm{H}_{2} \mathrm{O}$ ratios in these cold regions are found to be $(1.8-3.1) \times 10^{-3}$ and are consequently higher than in the hot inner regions of these sources.
\end{abstract}

Keywords: ISM: molecules-abundances-HDO, stars: protostars - hot cores 
KULCZAK

\section{INTRODUCTION}

During the cold phase preceding the formation of stellar objects, molecules freeze-out onto dust grains, forming $\mathrm{H}_{2} \mathrm{O}$ - dominated ice mantles, mixed with other less-abundant species. The low temperature and the disappearance of most molecules, especially $\mathrm{CO}$, from the gas phase trigger a peculiar chemistry leading to high abundances of deuterated species. Molecules tend to attach a D atom rather than an $\mathrm{H}$ atom, because deuterated species have larger reduced masses and consequently lower binding energies, arising from lower zero-point vibrational energies. Ion-molecule reactions in the gas phase (Brown \& Millar 1989) and reactions on the grain surfaces (Tielens 1983) are the two possible mechanisms responsible for deuterium enrichments in heavy molecules. The reactions involved are exothermic, which is why significant deuteration levels can be expected in the cold ISM. In the warmer phase, only very little fractionation is expected to occur, because the energy barrier could be overcome by the elevated temperature. However, at the temperatures deduced for the hot core regions (100-200 K) the ice mantles evaporate and the gas again becomes enriched in deuterated species, with abundances elevated compared to the cosmic $\mathrm{D} / \mathrm{H}$ ratio for a short period, before the chemistry reaches steady-state. These enhancements in the hot cores reflect, to some degree, the grain mantle composition in the earlier, colder cloud phases. Although the processes leading to the water deuteration are not fully understood, they are clearly related to the grain-surface chemistry and the observed $\mathrm{HDO} / \mathrm{H}_{2} \mathrm{O}$ ratio reveals the chemical and physical history of the protostellar materials (Cazaux et al. 2011; Codella et al. 2010). A recent review of water chemistry can be found in van Dishoeck et al. (2013) and a review of the latest observational results from Herschel.

Early studies of water deuterium fractionation in high-mass hot cores were performed almost twenty years ago, when the submillimeter spectrum was largely inaccessible (Jacq et al. 1990; Schulz et al. 1991: Gensheimer et al. 1996; Helmich et al. 1996). The HDO abundance has been recently determined toward the high-mass hot core G34.26+0.15 and the intermediate-mass protostar NGC7129 FIRS2 (Fuente et al. 2012; Liu et al. 2013; Coutens et al. 2014). Here, we present the new observations of the ground state rotational transition of $\mathrm{HDO}$ at $464.925 \mathrm{GHz}$ and the first excited transition at $509.292 \mathrm{GHz}$ toward three high-mass star forming regions: G34.26+0.15, W51 $\mathrm{e}_{1} / \mathrm{e}_{2}$ and $\mathrm{W} 49 \mathrm{~N}$. The combination of data taken with the same telescope of both the ground state transition $\left(1_{0,1}-0_{0,0}\right)$ and the first excited line $\left(1_{1,0}-1_{0,1}\right)$ provide better constraints on the source structure. These HDO transitions are studied for the first time to probe the structure of the envelope of the $W 51 \mathrm{e}_{1} / \mathrm{e}_{2}$ and $\mathrm{W} 49 \mathrm{~N}$ hot cores. In this paper, we aim at determining the HDO fractional abundances relative to $\mathrm{H}_{2}$ in the inner and outer region of the core in our target sources using, the static radiative transfer code of Zmuidzinas et al. (1995). We also used two $\mathrm{H}_{2}^{18} \mathrm{O}$ fundamental transitions observed by Flagey et al. (2013) to constrain the $\mathrm{H}_{2} \mathrm{O}$ abundance and the $\mathrm{HDO} / \mathrm{H}_{2} \mathrm{O}$ ratio in the outer envelopes.

\section{OBSERVATIONS}

Water is difficult to study from the ground, due to its strong presence in the Earth's atmosphere. However, many HDO lines, including the ground state $1_{0,1}-0_{0,0}$ rotational transition and the $1_{1,0}-1_{0,1}$ excited transition studied here, lie in atmospheric windows, where observations are possible from high sites, under good weather conditions. Table 1 lists the HDO transitions included in the present study and the $\mathrm{H}_{2}^{18} \mathrm{O}$ transitions which we use to constrain the $\mathrm{HDO} / \mathrm{H}_{2} \mathrm{O}$ ratio in the outer envelope.

\subsection{Source description}


Table 1. Observed and modeled $\mathrm{HDO}$ and $\mathrm{H}_{2}^{18} \mathrm{O}$ rotational transitions.

\begin{tabular}{|c|c|c|c|c|c|c|}
\hline Species & Transition & $\begin{array}{l}\text { Frequency } \\
(\mathrm{GHz})\end{array}$ & $\begin{array}{l}E_{\text {up }} \\
(\mathrm{K})\end{array}$ & $\begin{array}{c}A_{\mathrm{ul}} \\
\left(\mathrm{s}^{-1}\right)\end{array}$ & Telescope & $\begin{array}{c}\text { FWHM } \\
\left({ }^{\prime \prime}\right)\end{array}$ \\
\hline \multirow[t]{6}{*}{$\mathrm{HDO}$} & $1_{0,1}-0_{0,0}$ & 464.925 & 22.3 & $1.69 \times 10^{-4}$ & $\mathrm{CSO}$ & 15.7 \\
\hline & $1_{1,0}-1_{0,1}$ & 509.292 & 47.0 & $2.30 \times 10^{-3}$ & $\mathrm{CSO}$ & 15.7 \\
\hline & $2_{1,1}-2_{1,2}$ & 241.562 & 95.2 & $1.18 \times 10^{-5}$ & IRAM & 12.0 \\
\hline & $3_{1,2}-2_{2,1}$ & 225.897 & 167.6 & $1.31 \times 10^{-5}$ & IRAM & 13.0 \\
\hline & $4_{2,2}-4_{2,3}$ & 143.727 & 319.2 & $2.80 \times 10^{-6}$ & IRAM & 17.0 \\
\hline & $5_{2,3}-4_{3,2}$ & 255.050 & 437.2 & $1.78 \times 10^{-5}$ & IRAM & 12.0 \\
\hline $\mathrm{p}-\mathrm{H}_{2}^{18} \mathrm{O}$ & $1_{1,1}-0_{0,0}$ & 1101.698 & 53.9 & $1.79 \times 10^{-2}$ & HIFI & 19.2 \\
\hline $\mathrm{o}-\mathrm{H}_{2}^{18} \mathrm{O}$ & $2_{1,2}-1_{0,1}$ & 1655.868 & 114.0 & $1.26 \times 10^{-2}$ & HIFI & 12.8 \\
\hline
\end{tabular}

Note- Spectroscopic data from the JPL spectral line catalog (Pickett et al. 1998), http://www.spec.jpl.nasa.gov.

CSO data: this work; IRAM data from Jacq et al. (1990); Herschel/HIFI data from Flagey et al. (2013).

The $1_{0,1}-0_{0,0}$ ground-state rotational transition of $\mathrm{HDO}$ at $465 \mathrm{GHz}$ and the $1_{1,0}-1_{0,1}$ transition at $509 \mathrm{GHz}$ were observed toward three high-mass star forming regions are listed in Table 2. All the sources are characterized by strong millimeter continuum and mid-infrared emission, characteristic of the early stage of high-mass star formation.

G34.26+0.15 is one of the best studied high-mass star-forming regions in the Milky Way. Embedded within this molecular cloud is a hot core, which exhibits strong $\mathrm{H}_{2} \mathrm{O}$ maser emission and high abundances of saturated molecules (MacDonald et al. 1996); two unresolved UCHII regions, labeled $\mathrm{A}$ and B; a more evolved H II region with a cometary shape; and an extended, ring-like H II region (Reid \& Ho 1985). Based on narrow-band mid-infrared imaging of the complex, Campbell et al. (2000) concluded that the same star is responsible for ionization of the cometary H II component (C) and heating the dust, but is not interacting with the hot core seen in the molecular emission. At a $12^{\prime \prime}$ resolution, Hunter et al. (1998) also found the peak of the $350 \mu \mathrm{m}$ emission to coincide with the component $\mathrm{C}$ of the UCHII region.

The radio continuum emission of W51 shows three separate components: W51 IRS1, W51 IRS2, and W51 Main. W51 Main is defined by a group of $\mathrm{OH}$ and $\mathrm{H}_{2} \mathrm{O}$ masers near several UCHII regions. The continuum emission from ultracompact H II regions was resolved into compact components labeled $\mathrm{W} 51 \mathrm{e}_{1}$ to $\mathrm{W} 51 \mathrm{e}_{8}$ (Zhang \& Ho 1997). Of these, $\mathrm{e}_{1}$ and $\mathrm{e}_{2}$ are the brightest in high-resolution continuum maps.

The star forming region W49N contains at least a dozen UCHII regions powered by OB-type stars arranged in a ring 2 pc in diameter (De Pree et al. 2000). Evidence that star formation is still in progress within W49N comes from strong $\mathrm{H}_{2} \mathrm{O}$ maser emission and strong millimeter continuum emission attributed to dust condensations (Sievers et al. 1991).

\subsection{Observations}


Table 2. Source sample.

\begin{tabular}{|c|c|c|c|}
\hline Source & $\alpha(\mathrm{J} 2000)$ & $\delta(\mathrm{J} 2000)$ & $\begin{array}{c}D \\
(\mathrm{kpc})\end{array}$ \\
\hline $\mathrm{G} 34.26+0.15$ & 185318.6 & +011457.7 & $3.8^{\mathrm{a}}$ \\
\hline $\mathrm{W} 51 \mathrm{e}_{1} / \mathrm{e}_{2}$ & 192343.9 & +143025.9 & $5.4^{\mathrm{b}}$ \\
\hline W49N & 191013.2 & +090612.0 & $11.4^{\mathrm{c}}$ \\
\hline \multicolumn{4}{|c|}{ Fish et al. (2003). } \\
\hline \multicolumn{4}{|c|}{ Sato et al. (2010). } \\
\hline${ }^{\complement}$ Gwinn et al. & & & \\
\hline
\end{tabular}

Observations of the $465 \mathrm{GHz}$ and $509 \mathrm{GHz} \mathrm{HDO}$ transitions presented here were carried out in 2012 June-August, using the $10.4 \mathrm{~m}$ Leighton Telescope of the Caltech Submillimeter Observatory (CSO) on Mauna Kea, Hawaii. We used the new wideband $460 \mathrm{GHz}$ facility SIS receiver and the FFTS backend that covers the full $4 \mathrm{GHz}$ intermediate frequency (IF) range with a $270 \mathrm{kHz}$ channel spacing. Pointing of the telescope was checked by performing five-point continuum scans of planets and strong dust continuum sources. The spectra were obtained in antenna temperature units $T_{A}^{*}$, and then converted to the main beam brightness temperature, $T_{\mathrm{mb}}$, via the relation $T_{A}^{*}=\eta_{\mathrm{mb}} T_{\mathrm{mb}}, \eta_{\mathrm{mb}}$ is the CSO main-beam efficiency, which is found to be $\sim 37 \%$ at $460 \mathrm{GHz}$ from total power observations of planets. The absolute calibration uncertainty of the individual measurements is $\sim 20 \%$.

In addition to the new CSO data, we included in our analysis previously published observations of higher-energy transitions (Jacq et al. 1990) toward our target sources. We used the reduced HIFI data of the $\mathrm{H}_{2}^{18} \mathrm{O}$ transitions at 1101.698 and $1655.868 \mathrm{GHz}$. The $\mathrm{HDO}$ and $\mathrm{H}_{2}^{18} \mathrm{O}$ lines parameters are listed in Table 3. The $\mathrm{H}_{2}^{18} \mathrm{O}$ ground state transitions have been previously presented by Flagey et al. (2013).

The data processing was done using the IRAM GILDAS software package $($ Pety 2005$)$. We measured the line parameters: central velocity $V_{L S R}$, the full-width at half maximum (FWHM) $\Delta V$, peak intensity $T^{\text {peak }}$, by fitting a single gaussian profile to the data (in $T_{\mathrm{mb}}$ units). The integrated line intensity is equal to $W_{i}=\int T_{i} d V$, where $i$ either refers to observations (obs) or models (mod).

We also used Herschel/HIFI data at $893 \mathrm{GHz}$ (Vastel et al. in preparation) obtained by the PRISMAS guaranteed time key program, and the SCUBA data at $353 \mathrm{GHz}$ that provide an accurate determination of the source continuum flux.

The PRISMAS continuum observations were obtained in the double beam switching mode. At 800 $\mathrm{GHz}$, the HIFI beam size is 26.5 " and the instrument gain is $469 \mathrm{Jy} / \mathrm{K}$ (Roelfsema et al. 2012).

\section{DETERMINATION OF THE HDO AND $\mathrm{H}_{2} \mathrm{O}$ ABUNDANCE}

\subsection{Modeling}

The goal of this study is to determine the HDO fractional abundance in three high-mass stars formation regions. To reproduce the observed line intensities (Table 3) the static radiative transfer code of Zmuidzinas et al. (1995) is used. The model cloud is divided into 200 radial shells, and the code uses a multilevel accelerated lambda-iteration method (Rybicki \& Hummer 1991) to solve for the HDO level populations and the line and continuum radiative transfer in a self-consistent fashion. This radiative transfer program takes into account the excitation of HDO molecules by collisions, line 
Table 3. $\mathrm{HDO}$ and $\mathrm{H}_{2}^{18} \mathrm{O}$ lines parameters.

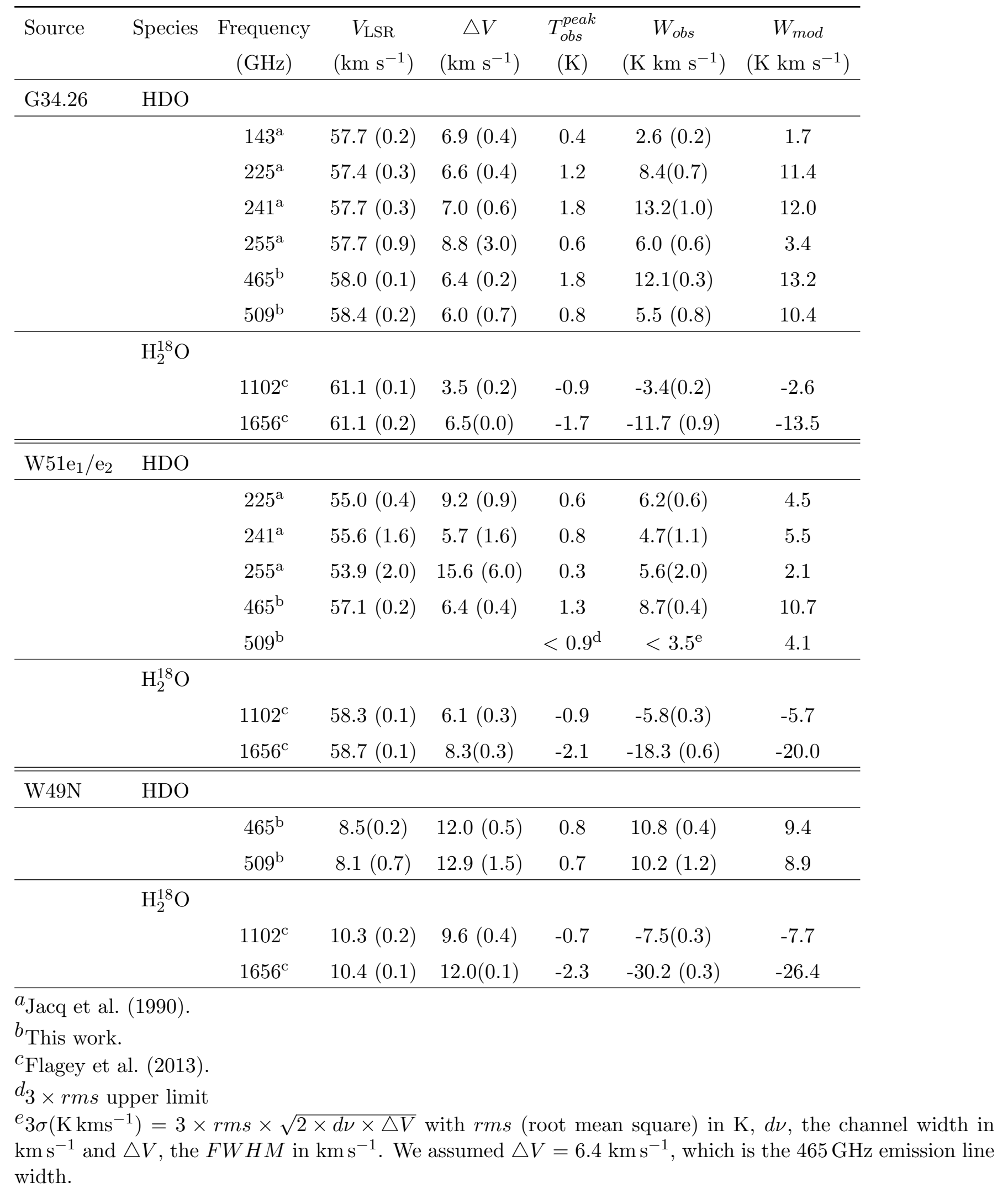


radiation, and dust continuum radiation at the HDO line frequencies. However, IR radiative pumping through HDO vibrationally excited levels and the large-scale velocity field, characteristic of infall or expansion, are not included. The HDO collisional rates used in this study were recently computed by Faure et al. (2012) and Wiesenfeld et al. (2011) for ortho- $\mathrm{H}_{2}$ and para- $\mathrm{H}_{2}$ in the temperature range 5-300 $\mathrm{K}$ and for all rotational transitions with an upper energies less than $444 \mathrm{~K}$. In the modelling, we assumed a constant ortho-to-para ratio (OPR) of $\mathrm{H}_{2}$ equal to 3. The ortho and para $\mathrm{H}_{2} \mathrm{O}$ collisional rates with ortho and para $\mathrm{H}_{2}$ were taken from the LAMDA data base (Schröier et al. 2005: Daniel et al. 2011). These rates were calculated for temperatures in the range from 5 to $1500 \mathrm{~K}$ including energy levels up to $2000 \mathrm{~K}$ above ground. The same collisional rates are used for the $\mathrm{H}_{2}^{18} \mathrm{O}$ isotopologue. The limitation imposed by the radiative transfer code is to use a single collisional partner in the calculations. We assume that all hydrogen is in the ortho state.

We carried out model calculations from the inner core radius, $r_{\min }$, to the outer radius, $r_{\max }$ (with

$r_{\text {max }} / r_{\text {min }} \sim 100$ for all sources; Hatchell \& van der Tak 2003). The distance of the edge of the core from the star is set at $r_{\text {min }} \sim 2.0 \times 10^{16} \mathrm{~cm}$ or $\sim 1000 \mathrm{AU}$, with no dust emission seen at the smaller radii. The lack of submillimeter emission in the core centers could be due to optical depth effects or a central cavity (van der Tak et al. 2000). We adopted a dust-to-gas ratio of 1:100 and a power-law $\mathrm{H}_{2}$ density distribution of the form:

$$
n(r)=n_{0}\left(\frac{r}{r_{\min }}\right)^{-1.5}
$$

where $n_{0}$ is the $\mathrm{H}_{2}$ density at the reference radius $\left(r_{\min }=1000 \mathrm{au}\right)$. The power-law index was set to 1.5 in accordance with the static infall theory in the inner part of the object (Shu 1977; van der Tak et al. 2000; Beuther et al. 2002; Marseille et al. 2010). We assumed that the gas and dust radial temperature profiles follow a power law (Viti \& Williams 1999):

$$
T(r)=T_{0}\left(\frac{r}{r_{\min }}\right)^{-0.5}
$$

where $T_{0}$ represents the maximum temperature of dust grains. We assumed that, at densities found in the hot cores, the gas temperature is equal to the dust temperature.

\subsection{Dust emissivity index $\beta$}

When molecules deplete inside prestellar cores, dust emission may represent the best tracer of the gas density distribution just prior to the onset of gravitational collapse. The dust continuum optical depth is described by a power-law frequency dependence, $\tau \propto \nu^{\beta}$, and to fit the observed spectral energy distribution, the knowledge of the grain emissivity spectral index, $\beta$, is required. The dust emissivity index depends on the dust grain composition, size, and temperature (Hildebrand 1983; Goldsmith et al. 1997). Details of the dust-modelling process can be found in the reviews by Draine (2003). Observationally, there have been many attempts at determining and explaining $\beta$. Typical values of $\beta$ range between 1 and 2, with further support for $\beta=1.5-2.0$ coming from observations: Wright et al. (1992), Minier et al. (2005) and Gordon et al. (2010). Planck Collaboration XIV (2013) used Planck HFI data with ancillary radio data to study the emissivity index. They computed a median value of far infrared spectral index $\beta_{F I R}=1.88 \pm 0.08$ at the high frequency Planck channels $(\nu \geq 353 \mathrm{GHz})$ and a median value of spectral index $\beta_{m m}=1.6 \pm 0.06$ at millimeter 
Table 4. Continuum flux densities and grain emissivity exponents

\begin{tabular}{lcccc}
\hline Source & $\begin{array}{c}F_{353}{ }^{\star \mathrm{a}} \\
(\mathrm{Jy} / \text { beam })\end{array}$ & $\begin{array}{c}F_{509}{ }^{\mathrm{b}} \\
(\mathrm{Jy} / \text { beam })\end{array}$ & $\begin{array}{c}F_{893}{ }^{\mathrm{b}} \\
(\mathrm{Jy} / \text { beam })\end{array}$ & $\beta$ \\
\hline G34.26+0.15 & 56.1 & 310 & 1320 & $1.6^{\mathrm{c}}$ \\
$\mathrm{W} 51 \mathrm{e}_{1} / \mathrm{e}_{2}$ & & 400 & 1490 & $1.7^{\mathrm{d}}$ \\
$\mathrm{W} 49 \mathrm{~N}$ & & 320 & 1450 & $1.8^{\mathrm{e}}$ \\
\hline
\end{tabular}

${ }^{\star} F_{\nu}$ the source flux density, $\nu$ the frequency of the observations in $\mathrm{GHz}$

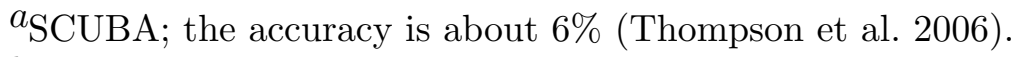

${ }^{b}$ Herschel; the accuracy is about $10 \%$.

${ }^{c}$ SIMBA and SCUBA observations (Hill et al. 2006).

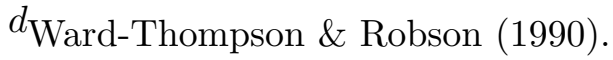

${ }^{e}$ Gordon et al. (1987).

wavelengths $(\nu<353 \mathrm{GHz})$. We can estimate the dust grain emissivity exponent from observations at two frequencies $\nu_{1}$ and $\nu_{2}$ (Hill et al. 2006):

$$
\beta=\frac{\log \frac{F_{\nu_{2}}}{F_{\nu_{1}}}+\log \frac{\left(\mathrm{e}^{h \nu_{2} / k T_{\text {dust }}}-1\right)}{\left(\mathrm{e}^{h \nu_{1} / k T_{\text {dust }}}-1\right)}}{\log \frac{\nu_{2}}{\nu_{1}}}-3
$$

where $F_{\nu}$ is the source flux density, $\nu$ the frequency of the observations, and $T_{\text {dust }}$ the dust temperature. In this work, the dust grain emissivity index $\beta$ is determined for G34.26 using the millimeter $\left(\lambda_{1}=1.2 \mathrm{~mm} ; \nu_{1}=250 \mathrm{GHz}\right)$ data obtained with SIMBA and submillimeter $\left(\lambda_{2}=450 \mu \mathrm{m} ; \nu_{2}=\right.$ $660 \mathrm{GHz}$ ) SCUBA data. We derive $\beta=1.6 \pm 0.48$. The uncertainty in $\beta$ is typically $30 \%$ for the 20-50 K temperature range (Hill et al. 2006). The spectral index for the other sources is taken from literature: Ward-Thompson \& Robson (1990) for W49N and from Gordon \& Jewell (1987) for W51. The HDO lines studied here are seen in emission, and the model intensities are not sensitive to the exact value of $\beta$, especially for $T>30 \mathrm{~K}$. That is why the dust grain emissivity index is fixed, and not a free parameter, in the fits.

The values of $\beta$ in our target sources, as well as the continuum fluxes from SCUBA and Herschel/HIFI observations, are listed in Table 4. These are used to constrain the density and temperature distributions as input to the line modeling.

\subsection{Modeling procedure}

We approximated the radial variation of the HDO fractional abundance, $X=n(H D O) / n\left(H_{2}\right)$ as a step function with an enhanced abundance $X_{i n}$ in the inner region where $T \geq 100 \mathrm{~K}$, and a lower value $X_{\text {out }}$ for the outer envelope where $T<100 \mathrm{~K}$. Laboratory studies indicate that the evaporation temperature lies in the 90-110 K range, depending on the ice composition and structure (Fraser et al. 2005). In this work the sublimation temperature of water, $T=100 \mathrm{~K}$ (Fraser et al. 2001), is applied as the jump temperature. The model uses the following free parameters: $n_{0}, T_{0}$ and $X_{\text {in }}$ (for $T \geq 100 \mathrm{~K}$ ) and $X_{\text {out }}$ (for $T<100 \mathrm{~K}$ ). We determine the continuum flux density per beam at 353 $\mathrm{GHz}(850 \mu \mathrm{m}), 509 \mathrm{GHz}(590 \mu \mathrm{m})$, and $893 \mathrm{GHz}(336 \mu \mathrm{m})$ from the model.

Finally, the synthetic spectra and continuum emission are convolved to the appropriate telescope beam size for comparison with the observations. We minimize the 'figure of merit' (FOM) with the 
method of Jacq et al. (1990) to find the best model of the source. The FOM is computed from the observed and modeled spectra and continuum fluxes according to the following formula:

$$
F O M=F O M_{1}+F O M_{2}=\sum_{n} \frac{\left(T_{\mathrm{obs}}-T_{\mathrm{mod}}\right)^{2}}{\left(T_{\mathrm{obs}}\right)^{2}}+\sum_{m} \frac{\left(F_{\mathrm{obs}}-F_{\mathrm{mod}}\right)^{2}}{\left(F_{\mathrm{obs}}\right)^{2}}
$$

for a set of $n$ spectral lines and $m$ continuum flux densities. The inner and outer HDO abundances are constrained by the spectral line data $\left(F O M_{1}\right)$, whereas model parameters describing the density and temperature distribution are constrained primarily by the continuum SED $\left(F O M_{2}\right)$. To determine the uncertainty of $X_{\text {in }}$ and $X_{\text {out }}$, we performed $\chi^{2}$ analysis. In analogy to Lampton method Lampton et al. 1976) we define $S \equiv F O M_{1} / \sigma^{2}=\sum_{n} \frac{\left(T_{\mathrm{obs}}-T_{\mathrm{mod}}\right)^{2}}{\left(\sigma T_{\mathrm{obs}}\right)^{2}}$, where $n$ being the number of spectral lines. The $\sigma$ within analysis $\chi^{2}$ includes a calibrations uncertainty of $20 \%$ for all individual measurments. The difference, $\Delta S \equiv S_{\text {true }}-S_{\text {min }}$ is distributed as $\chi^{2}$ with $p$ degrees of freedom (here: $p=2 ; X_{\text {in }}$ and $\left.X_{\text {out }}\right)$. By $\Delta S \sim \chi_{p}^{2}$ (" " - "is distributed as") we mean for any number L probability: $\operatorname{Prob}(\Delta S>L)=\operatorname{Prob}\left(\chi_{p}^{2}>L\right)$. With the limiting contour value $S_{L}$ defined as $S_{\min }+L$, $\operatorname{Prob}\left(\Delta S>S_{L}-S_{\text {min }}\right)=\operatorname{Prob}\left(S_{\text {true }}>S_{L}\right)=\operatorname{Prob}\left(\chi_{p}^{2}>S_{L}-S_{\text {min }}\right)$. Prob $\left(S_{\text {true }}>S_{L}\right)$ is the probality $\alpha$ of the contour failing to enclose the true value, hence $\alpha=\operatorname{Prob}\left(\chi_{p}^{2}>S_{L}-S_{\min }\right)$. The $\alpha$-point of $\chi^{2}$ distribution is defined by $\alpha \equiv \operatorname{Prob}\left[\chi_{p}^{2}>\chi_{p}^{2}(\alpha)\right]$. Significance $\alpha$ is $S_{L}=S_{\min }+\chi_{p}^{2}(\alpha)$. In this expression, $\chi_{p}^{2}(\alpha)$ is tabulated value of $\chi^{2}$ distribtion for $p$ degrees of freedom and significance $\alpha$. Equivalently, any one observation's contour has a confidence $C=1-\alpha$ of enclosing the true parameter vector. The required contours for significance are $1 \sigma, 2 \sigma$, and $3 \sigma$, which respectively represent a confidence of $68.3 \%, 95.4 \%$, and $99.7 \%$ of enclosing the true value of $X_{\text {in }}$ and $X_{\text {out }}$. The contours correspond to $S_{L}=S_{\min }+2.17, S_{L}=S_{\min }+6.17$, and $S_{L}=S_{\min }+11.8$.

We used models with the same physical parameters as those used for HDO in the analysis of the $\mathrm{H}_{2}^{18} \mathrm{O}$ data. The two $\mathrm{H}_{2}^{18} \mathrm{O}$ fundamental transitions were modeled independently and the resulting ortho/para ratio of water is consistent with the high temperature value of given the modeling uncertainties .

\subsection{Results}

\subsubsection{Origin of The Lines}

Figure 1 shows fractional population of the HDO levels of the relevant features of our dataset calculated in our model. The high-energy transitions are sensitive to changes in $X_{i n}$. Indeed the bulk of emission in the high energy transitions is produced in the inner hot-core region where $T>100 \mathrm{~K}$. In the G34.26 source, this region has a radius equal to $1.0^{\prime \prime}$ which corresponds to $0.02 \mathrm{pc}$. This is in agreement with the interferometric observations of the HDO lines at 225 and $241 \mathrm{GHz}$ by Liu et al. (2013). That is the reason why the high-energy transitions are sensitive to changes in $X_{i n}$. On the other hand, the 465 and $509 \mathrm{GHz}$ HDO transitions are sensitive primarily to $X_{\text {out }}$. The $509 \mathrm{GHz}$ line arises predominantly in the region between the warm envelope and the cold region $(T \sim 50-100 \mathrm{~K})$, whereas the $465 \mathrm{GHz}$ transition is produced in the cold envelope $(T<50 \mathrm{~K})$. The ground-state rotational transition of $\mathrm{HDO}$ at $465 \mathrm{GHz}$ is consequently a very good probe of the abundance in the cold outer envelope, which is consistent with the results of Parise et al. (2005) for the solar-type protostar IRAS 16293-2422. The $509 \mathrm{GHz}$ transition provides particurlarly good constraints on the HDO abundance profile in the transition region between the hot core and the envelope, and should be included in future, more advanced models of HDO in high-mass star-forming regions. The model 


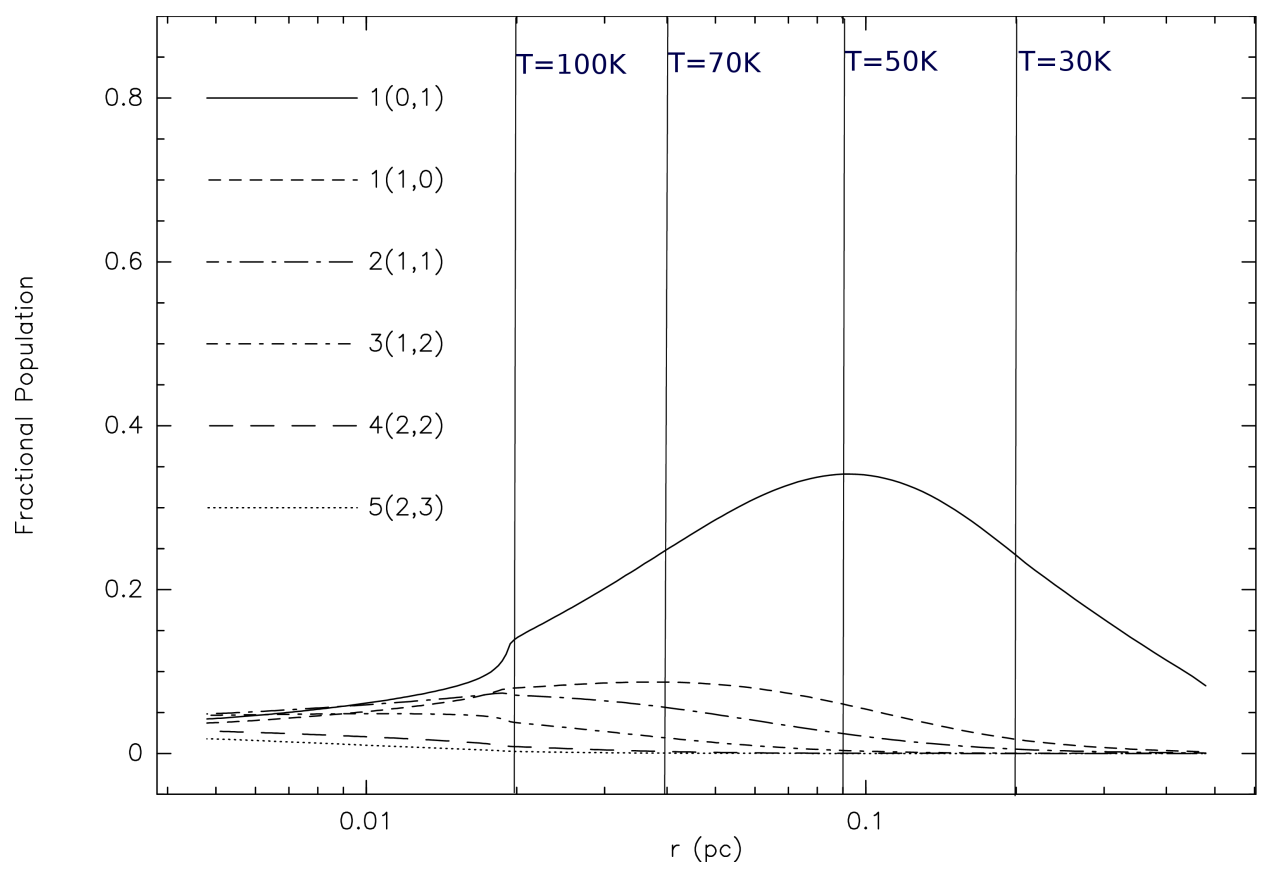

Figure 1. Fractional population of the HDO energy levels as a function of the radius of the cloud.

reproduces the observed intensities of different transitions in our target sources, with the exception of the $509 \mathrm{GHz}$ line in G34.26. Although the signal-to-noise ratio of the observed $509 \mathrm{GHz}$ spectrum is limited, it is clear that the best-fit model does not reproduce this line profile. The $509 \mathrm{GHz}$ transition is formed in the part of the cloud where, within the scenario proposed by Rolffs et al. (2010), various feedback: thermal, radiative, or turbulent mechanisms are expected during the process of massive stars formation. In particular, it should be noted that, with the inclusion of velocity fields in their model, Coutens et al. (2014) succesfully reproduced the $509 \mathrm{GHz}$ line observed with Herschel/HIFI toward this same source. Other possibility is an accretion disk that is fed by the infalling envelope. This is also supported by observations made by Keto et al. (1987) of G34.26. The size of this possible disk is about 9000 au (0.05 pc) (Garay \& Rodriguez 1990; Hajigholi et al. 2016) and agrees well with the place where the $509 \mathrm{GHz}$ line arises (see Figure 1). This model of the G34.26 source (Hajigholi et al. 2016) neither confirm or refute the presence of an expansion in the inner parts of the envelope (Coutens et al. 2014). We concluded that the geometry and physical structure of our model is too simplistic, and that is why we could not to reproduce the $509 \mathrm{GHz}$ line.

\subsubsection{Target sources}

G34.26+0.15: Observed spectra (black line) and gaussian fit (blue line) of the 465 and $509 \mathrm{GHz}$ HDO transitions toward G34.26+0.15 along with the best-fit model (red line) are shown in Figure 2.

Model results are presented in Table 5 . We obtain the best-fit model for: $T_{0}=200 \mathrm{~K}, n_{0}=$ $1.0 \times 10^{8} \mathrm{~cm}^{-3}, X_{\text {in }}=3.7 \times 10^{-8}$ and $X_{\text {out }}=7.8 \times 10^{-11}$. We calculated continuum flux densities at 353, 509, and $893 \mathrm{GHz}$. The resulting uncertainties of $X_{\text {in }}$ and $X_{\text {out }}$ are shown in Figure 3 and listed in Table 8.

Observed and modeled spectra of the para- $\mathrm{H}_{2}^{18} \mathrm{O}$ line at $1102 \mathrm{GHz}$ and the ortho- $\mathrm{H}_{2}^{18} \mathrm{O}$ line at $1656 \mathrm{GHz}$ are shown in Figure 2. The derived OPR in G34.26 is 1.9. The total (ortho+para) $\mathrm{H}_{2}^{18} \mathrm{O}$ abundance in the envelope $X_{\text {out }}$, is $4.9 \times 10^{-11}$. The recommended isotopic abundance ratio between 

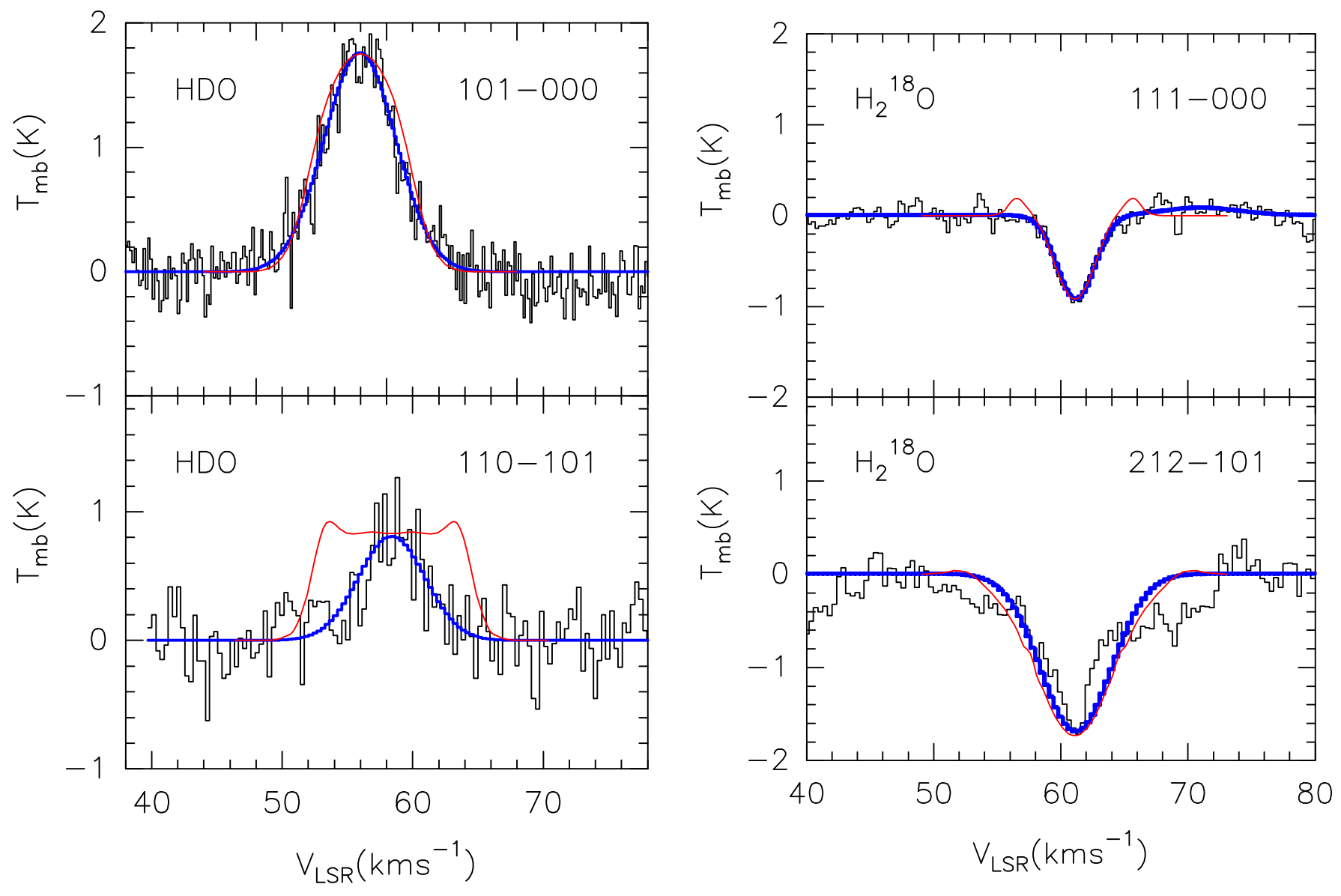

Figure 2. Observed spectra of the 465 and $509 \mathrm{GHz} \mathrm{HDO}$ lines and 1102 and $1656 \mathrm{GHz} \mathrm{H}_{2}^{18} \mathrm{O}$ lines towards G34.26+0.15. The Gaussian fits are shown in blue, while the best-fit model in red.

${ }^{16} \mathrm{O}$ and ${ }^{18} \mathrm{O}$ is 500 (Lodders 2003). Using this value, the $\mathrm{H}_{2} \mathrm{O}$ outer abundance is $2.5 \times 10^{-8}$ and the outer $\mathrm{HDO} / \mathrm{H}_{2} \mathrm{O}$ ratio is $3.1 \times 10^{-3}$ in the envelope. Considering the results with a $20 \%$ calibration uncertaint, the outer abundance ratio is $(2.5-3.7) \times 10^{-3}$.

$W 51 e_{1} / e_{2}$ : Observed spectra and gaussian fit of the 465 and $509 \mathrm{GHz}$ HDO transitions toward W51e $1 / e_{2}$ along with the best-fit model, are shown in Figure 4 by black, blue and red lines, respectively. Model results for W51 are presented in Table 6. We obtain the best fit for: $T_{0}=230 \mathrm{~K}, n_{0}=1.8 \times 10^{8} \mathrm{~cm}^{-3}, X_{\text {in }}=1.7 \times 10^{-8}$ and $X_{\text {out }}=7.0 \times 10^{-11}$. The resulting uncertainties of $X_{\text {in }}$ and $X_{\text {out }}$ are shown in Figure 5 and listed in Table 8.

Model flux densities per beam at $509 \mathrm{GHz}$ and $893 \mathrm{GHz}$ for W51 are also listed in Table 6.

Observed and modeled spectra of the para- $\mathrm{H}_{2}^{18} \mathrm{O}$ line at $1102 \mathrm{GHz}$ and the ortho- $\mathrm{H}_{2}^{18} \mathrm{O}$ line at $1656 \mathrm{GHz}$ are shown in Figure 4. The total $\mathrm{H}_{2}^{18} \mathrm{O}$ abundance $\left(\mathrm{OPR}=2.9\right.$ ) in the envelope $X_{\text {out }}$, is $5.5 \times 10^{-11}$. The $\mathrm{H}_{2} \mathrm{O}$ outer abundance is $2.8 \times 10^{-8}$ and outer $\mathrm{HDO} / \mathrm{H}_{2} \mathrm{O}$ ratio is $2.5 \times 10^{-3}$. Considering the results with the $20 \%$ calibration uncertainty the outer ratio is $(2.0-3.0) \times 10^{-3}$.

W49N: Observed spectra and gaussian fit of the 465 and $509 \mathrm{GHz}$ HDO transitions toward W49N along with the best-fit model are shown in Figure 6 by black, blue and red lines, respectively. Model 


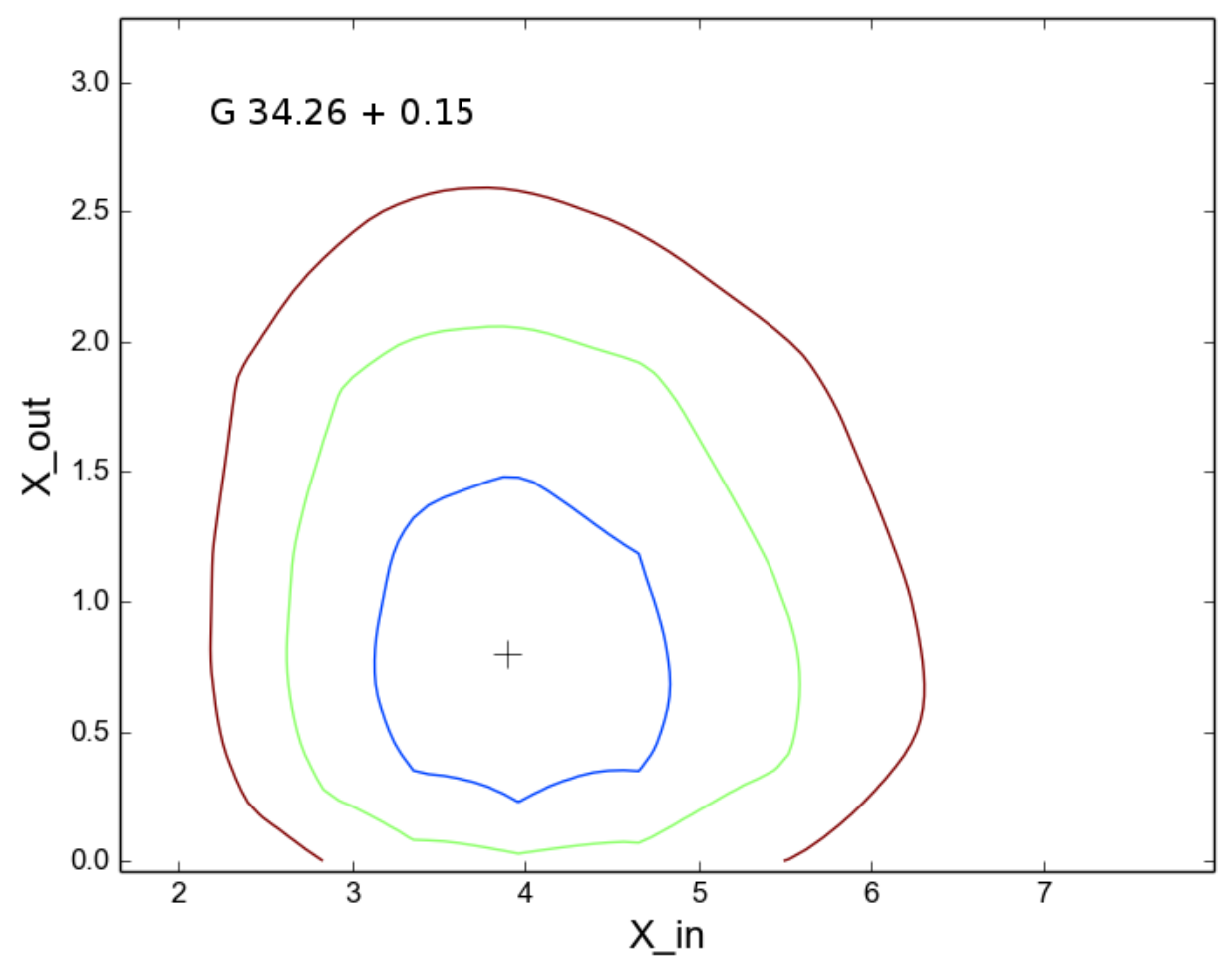

Figure 3. $X_{\text {in }}$ and $X_{\text {out }}$ HDO abundance contours at $1 \sigma, 2 \sigma, 3 \sigma$ for $\chi^{2}$. The best-fit model is represented by the symbol " $+"\left(X_{\text {in }}=\mathrm{a} \times 10^{-8}, X_{\text {out }}=\mathrm{b} \times 10^{-10}\right)$.

results for $\mathrm{W} 49 \mathrm{~N}$ are presented in Table 7 . We obtain the best fit for: $T_{0}=300 \mathrm{~K}, n_{0}=2.5 \times 10^{8} \mathrm{~cm}^{-3}$, $X_{\text {in }}=0.3 \times 10^{-8}$ and $X_{\text {out }}=10 \times 10^{-11}$. The resulting uncertainties of $X_{\text {in }}$ and $X_{\text {out }}$ are shown in Figure 7 and listed in Table 8.

As data on the high excitation lines are missing for W49N, the inner abundance $X_{\text {in }}$ is not as well constrained as in the other sources.

Observed and modeled spectra of the para- $\mathrm{H}_{2}^{18} \mathrm{O}$ line at $1102 \mathrm{GHz}$ and the ortho- $\mathrm{H}_{2}^{18} \mathrm{O}$ line at $1656 \mathrm{GHz}$ are shown in Figure 6. The total $\mathrm{H}_{2}^{18} \mathrm{O}(\mathrm{OPR}=3.1)$ abundance in the envelope $X_{\text {out }}$, is $1.1 \times 10^{-10}$. The $\mathrm{H}_{2} \mathrm{O}$ outer abundance is $5.5 \times 10^{-8}$ and the outer $\mathrm{HDO} / \mathrm{H}_{2} \mathrm{O}$ ratio is $1.8 \times 10^{-3}$. Considering the results with the $20 \%$ calibration uncertainty, the outer abundance ratio is (1.4 $2.2) \times 10^{-3}$.

\section{DISCUSSION}

\subsection{Comparison with Previous Studies}

Previous observations of the high-mass star-forming regions indicate an HDO abundance in the hot cores $(\mathrm{T}>100 \mathrm{~K})$ but not in the outer, cooler envelopes $(\mathrm{T}<100 \mathrm{~K})$ (Jacq et al. 1990, Gensheimer et al. 1996, Pardo et al. 2001; see Table 9). We could find variations in the derived HDO abundances in the hot cores between $1.5 \times 10^{-9}$ and $2.0 \times 10^{-7}$. Jacq et al. (1990) belived that, independent of all modeling, a value much lower than $3.0 \times 10^{-8}$ for $\mathrm{HDO} / \mathrm{H}_{2}$ in the hot core is very unlikely. For the first time, Comito et al. $(2003,2010)$ estimated the HDO abundance in both the inner and outer 
Table 5. Line intensities and continuum fluxes for the best fit model of G34.26+0.15.

\begin{tabular}{|c|c|c|c|c|}
\hline $\begin{array}{l}\text { HDO } \\
\text { transitions }\end{array}$ & $\begin{array}{c}\text { Freq } \\
(\mathrm{GHz})\end{array}$ & $\begin{array}{l}T_{\mathrm{obs}} \\
(\mathrm{K})\end{array}$ & $\begin{array}{c}T_{\text {mod }} \\
(\mathrm{K})\end{array}$ & $F O M_{1}$ \\
\hline $1_{0,1}-0_{0,0}$ & $464.925^{\mathrm{a}}$ & 1.8 & 1.8 & 0.00 \\
\hline $1_{1,0}-1_{0,1}$ & $509.292^{\mathrm{a}}$ & 0.8 & 0.8 & 0.00 \\
\hline $2_{1,1}-2_{1,2}$ & $241.562^{\mathrm{b}}$ & 1.8 & 1.6 & 0.01 \\
\hline $3_{1,2}-2_{2,1}$ & $225.897^{\mathrm{b}}$ & 1.2 & 1.4 & 0.03 \\
\hline $4_{2,2}-4_{2,3}$ & $143.727^{\mathrm{b}}$ & 0.4 & 0.5 & 0.06 \\
\hline $5_{2,3}-4_{3,2}$ & $255.050^{\mathrm{b}}$ & 0.6 & 0.4 & 0.11 \\
\hline \multirow[t]{7}{*}{ Flux (Jy/beam) } & $F_{353}{ }^{\mathrm{c}}$ & $F_{353_{\text {mod }}}$ & & $\mathrm{FOM}_{2}$ \\
\hline & 56.1 & 56.4 & & 0.00 \\
\hline & $F_{509}{ }^{\mathrm{d}}$ & $F_{509_{\text {mod }}}$ & & \\
\hline & 310 & 376 & & 0.05 \\
\hline & $F_{893}{ }^{\mathrm{d}}$ & $F_{893_{\text {mod }}}$ & & \\
\hline & 1320 & 1525 & & 0.02 \\
\hline & & & & $F O M=0.28$ \\
\hline
\end{tabular}

$a_{\text {This work. }}$

$b$ Jacq et al. (1990).

${ }^{c} \mathrm{CSO}$

$d_{\text {Herschel }}$

region of the high-mass source Sgr B2(M). These are, respectively: $3.5 \times 10^{-9}(\mathrm{~T}>200 \mathrm{~K}), 1.5 \times 10^{-9}$ $(100 \mathrm{~K}<\mathrm{T}<200 \mathrm{~K})$ and $2.5 \times 10^{-11}(\mathrm{~T}<100 \mathrm{~K})$. The singly deuterated form of water has been also observed in the massive source AFGL 2591, with abundance varying from $1 \times 10^{-7}$ in the hot core and $4 \times 10^{-9}$ in the outer envelope (van der Tak et al. 2006). Liu et al. (2013) and Coutens et al. (2014) determined the $\mathrm{HDO}$ abundance and $\mathrm{HDO} / \mathrm{H}_{2} \mathrm{O}$ ratio in the inner and outer region of G34.26 (see Table 9). We derived the HDO abundances of $X_{\text {in }}$ and $X_{\text {out }}$ in three high-mass star-forming regions: G34.26, W 49N, and W51. We found a difference between our $X_{i n}(H D O)$ and $X_{\text {out }}(H D O)$ values for G34.26 and those reported by Coutens et al. (2014) and Liu et al. (2013), respectively. This is likely because the first authors used different model structures and a higher jump temperature, and the second authors did not check the higher value of $X_{\text {out }}(H D O)$ in their model. The obtained HDO abundances of our target sources in the hot cores and the cooler envelopes are relatively consistent with the values found in the other high-mass star-forming regions (Kulczak-Jastrzȩbska 2016). These results show that the HDO abundance is enriched in the inner regions of high-mass protostars because of the sublimation of the ice mantles, in the same way as for other studies low- and high-mass sources (e.g. NGC 1333 IRAS2A, IRAS 162923-2422, AFGL 2591, G34.26; Table 9). Observations of sites of high-mass star formation show in general the lower HDO abundances than observations of lowmass star forming cores. Possibly for high-mass protostars, the very cold and dense pre-collapse phase where CO freeze-out onto the grain mantles lasts only a short time, and the chemical reactions 

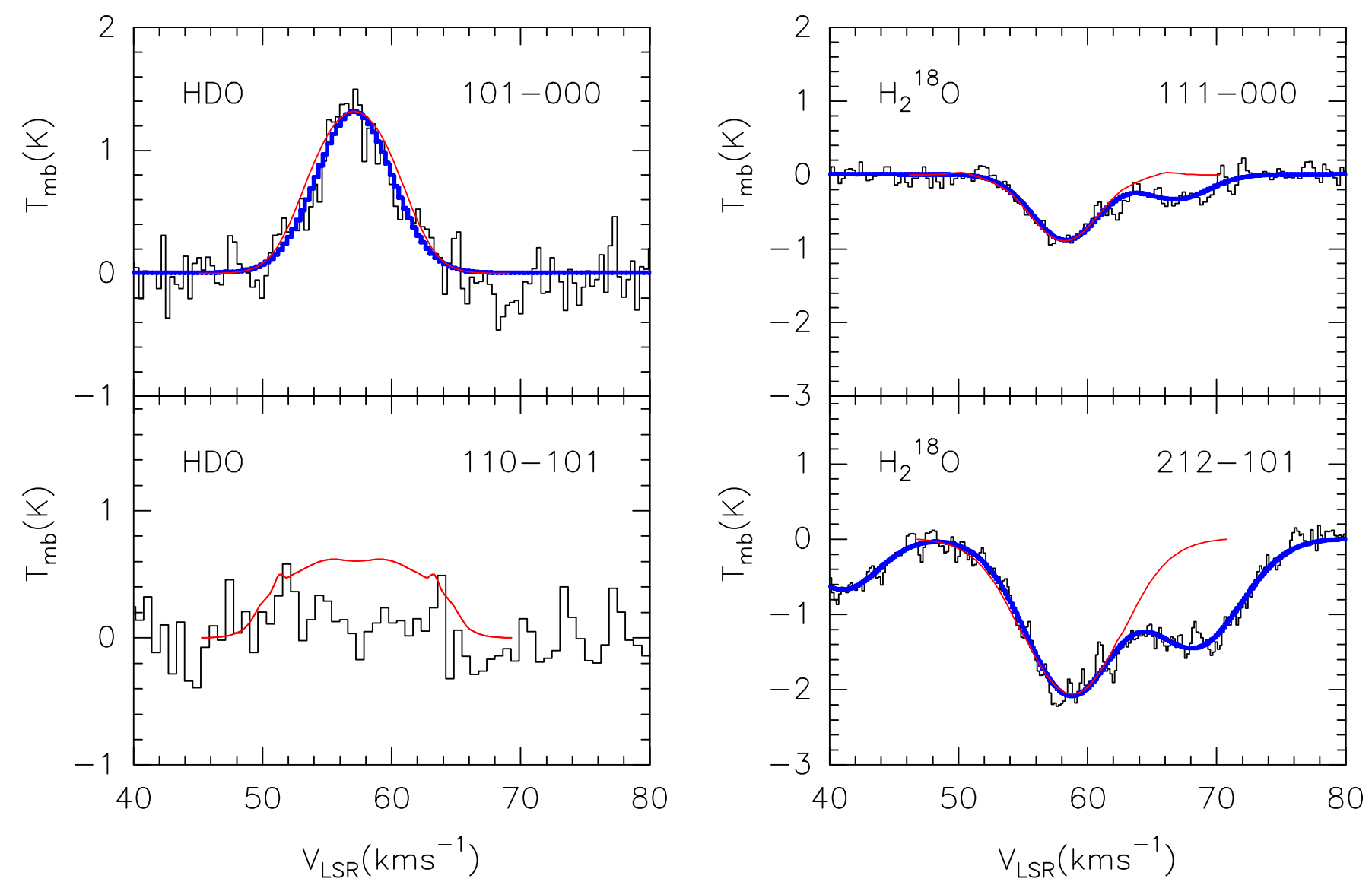

Figure 4. Observed spectra of the 465 and $509 \mathrm{GHz}$ HDO lines and 1102 and $1656 \mathrm{GHz} \mathrm{H}_{2}^{18} \mathrm{O}$ lines toward W51e $1 / e_{2}$.The Gaussian fits are shown in blue, while the best-fit model in red.

leading to the enhancement of deuterium abundance being strongly depressed when the temperature increases (Caselli et al. 2008).

\subsection{Variation of the $\mathrm{HDO} / \mathrm{H}_{2} \mathrm{O}$ Ratios with the Radius}

Based on observations of two $\mathrm{H}_{2}^{18} \mathrm{O}$ fundamental transitions, we found that the $\mathrm{H}_{2} \mathrm{O}$ abundances in our target sources are $(2.5-5.5) \times 10^{-8}$. Similar values were found for the other high-mass protostars: $5 \times 10^{-10}-4 \times 10^{-8}$ (Marseille et al. 2010; Herpin et al. 2012; van der Tak et al. 2010; Choi et al. 2015). The $\mathrm{H}_{2} \mathrm{O}$ abundance in the cold envelope agrees fairly well with the model predictions for cold regions where freeze-out takes place (Ceccarelli et al. 1996; van der Tak et al. 2013).

The water-deuterium fractionation in the inner and outer envelope of the high-mass star-forming region G34.26 was previously estimated by Liu et al. (2013) and Coutens et al. (2014). We determined the outer $\mathrm{HDO} / \mathrm{H}_{2} \mathrm{O}$ ratio in $\mathrm{G} 34.26$ to be $3.1 \times 10^{-3}$, this value is relatively consistent with Coutens et al. (2014) (see Table 9). To estimate the inner $\mathrm{HDO} / \mathrm{H}_{2} \mathrm{O}$ ratio for the target sources, we used an inner $\mathrm{H}_{2} \mathrm{O}$ abundance value as high as $10^{-4}$ from observations of other high-mass star formingregions (Boonman 2003; Snell et al. 2000; Chavarría et al. 2010; Herpin et al. 2012; van der Tak et al. 2013). However a lower value of $\sim 10^{-6}$ was found in NGC 6334 I (Emprechtinger et al. 2013). A possible explanation for the low water abundance in this source is a time-dependent effect; water 


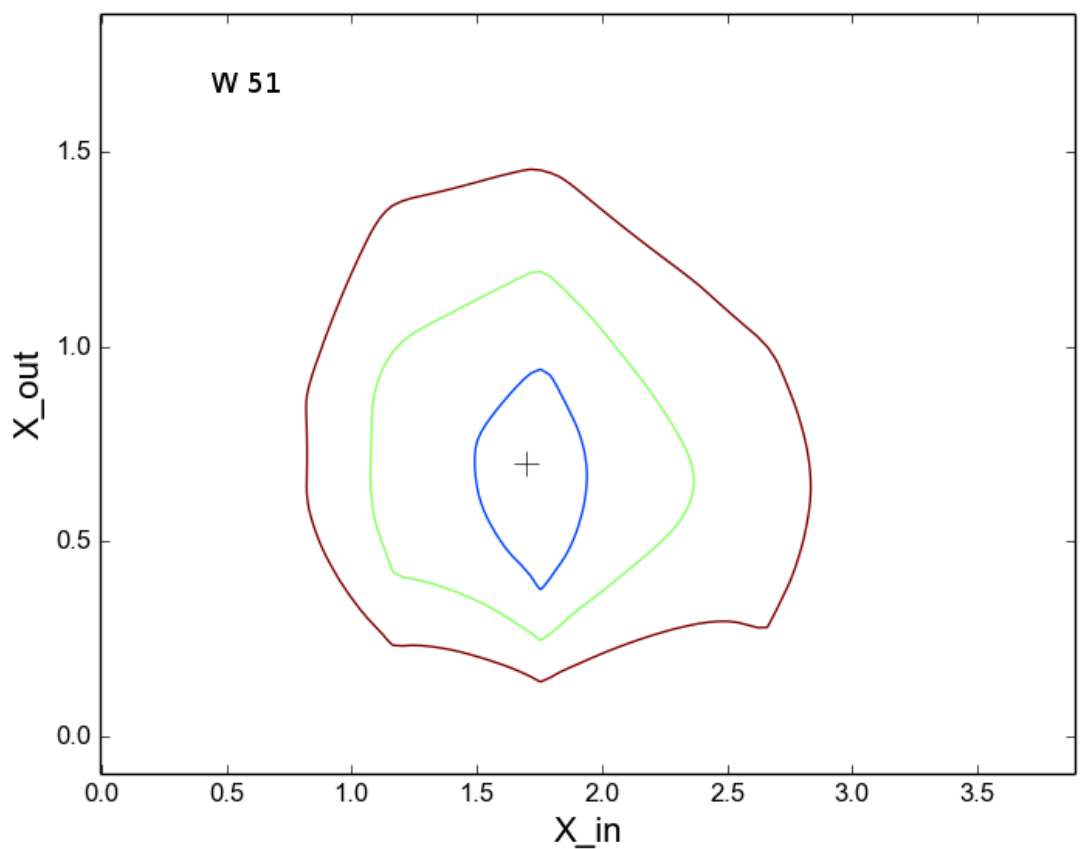

Figure 5. $X_{\text {in }}$ and $X_{\text {out }}$ HDO abundance contours at $1 \sigma, 2 \sigma, 3 \sigma$ for $\chi^{2}$. The best-fit model is represented by the symbol " $"\left(X_{\text {in }}=\mathrm{a} \times 10^{-8}, X_{\text {out }}=\mathrm{b} \times 10^{-10}\right)$.

Table 6. Line intensities and continuum fluxes for the best fit model of W51e $1 / \mathrm{e}_{2}$.

\begin{tabular}{lcccc}
\hline $\begin{array}{l}\text { HDO } \\
\text { transitions }\end{array}$ & $\begin{array}{c}\text { Freq } \\
(\mathrm{GHz})\end{array}$ & $\begin{array}{c}T_{\text {obs }} \\
(\mathrm{K})\end{array}$ & $\begin{array}{c}T_{\text {mod }} \\
(\mathrm{K})\end{array}$ & FOM \\
\hline $1_{0,1}-0_{0,0}$ & $464.925^{\mathrm{a}}$ & 1.3 & 1.3 & 0.00 \\
$2_{1,0}-1_{0,1}$ & $509.292^{\mathrm{a}}$ & $<0.9$ & 0.6 & \\
$2_{1,1}-2_{1,2}$ & $241.562^{\mathrm{b}}$ & 0.8 & 0.8 & 0.00 \\
$3_{1,2}-2_{2,1}$ & $225.897^{\mathrm{b}}$ & 0.6 & 0.67 & 0.01 \\
$5_{2,3}-4_{3,2}$ & $255.050^{\mathrm{b}}$ & 0.3 & 0.2 & 0.11 \\
\hline \hline Flux $(\mathrm{Jy} /$ beam $)$ & $F_{509} \mathrm{c}$ & $F_{509}$ & & $F O M_{2}$ \\
& 400 & 350 & & 0.02 \\
\hline & $F_{893} \mathrm{c}$ & $F_{893_{\bmod }}$ & & \\
& 1490 & 1509 & & 0.00 \\
& & & & $F O M=0.14$ \\
\hline
\end{tabular}

$a_{\text {This work. }}$

$b$ Jacq et al. (1990).

${ }^{c}$ Herschel

molecules may not have enough time to fully desorp from the dust grain. Our derived $\mathrm{HDO} / \mathrm{H}_{2} \mathrm{O}$ 

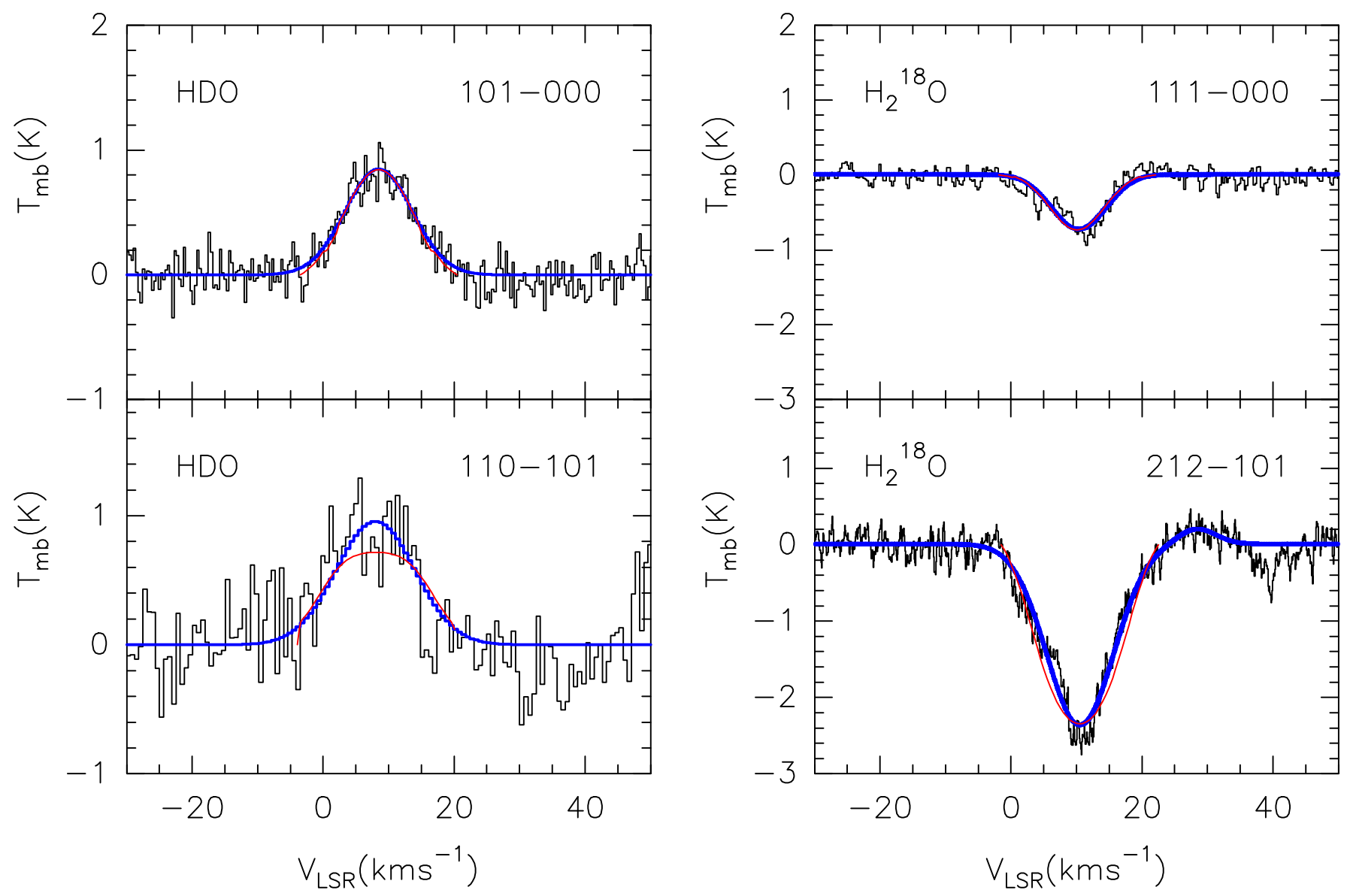

Figure 6. Observed spectra of the 465 and $509 \mathrm{GHz}$ HDO lines and 1102 and $1656 \mathrm{GHz} \mathrm{H}_{2}^{18} \mathrm{O}$ lines toward W49N. The Gaussian fits are shown in blue, while the best-fit model in red.

Table 7. Line intensities and continuum fluxes for the best fit model of W49N.

\begin{tabular}{lcccc}
\hline $\begin{array}{l}\text { HDO } \\
\text { transitions }\end{array}$ & $\begin{array}{c}\text { Freq } \\
(\mathrm{GHz})\end{array}$ & $\begin{array}{c}T_{\text {obs }} \\
(\mathrm{K})\end{array}$ & $\begin{array}{c}T_{\text {mod }} \\
(\mathrm{K})\end{array}$ & FOM \\
\hline $1_{0,1}-0_{0,0}$ & $464.925^{\mathrm{a}}$ & 0.8 & 0.8 & 0.00 \\
$2_{1,0^{-}-1_{0,1}}$ & $509.292^{\mathrm{a}}$ & 0.7 & 0.6 & 0.02 \\
\hline \hline Flux (Jy/beam) & $F_{509} \mathrm{~b}$ & $F_{509_{\bmod }}$ & $F O M_{2}$ \\
& 320 & 310 & & 0.00 \\
\hline & $F_{893} \mathrm{~b}$ & $F_{893_{\bmod }}$ & \\
& 1450 & 1410 & & 0.01 \\
& & & & $F O M=0.03$ \\
\hline
\end{tabular}

$a_{\text {This work. }}$

$b_{\text {Herschel }}$ 


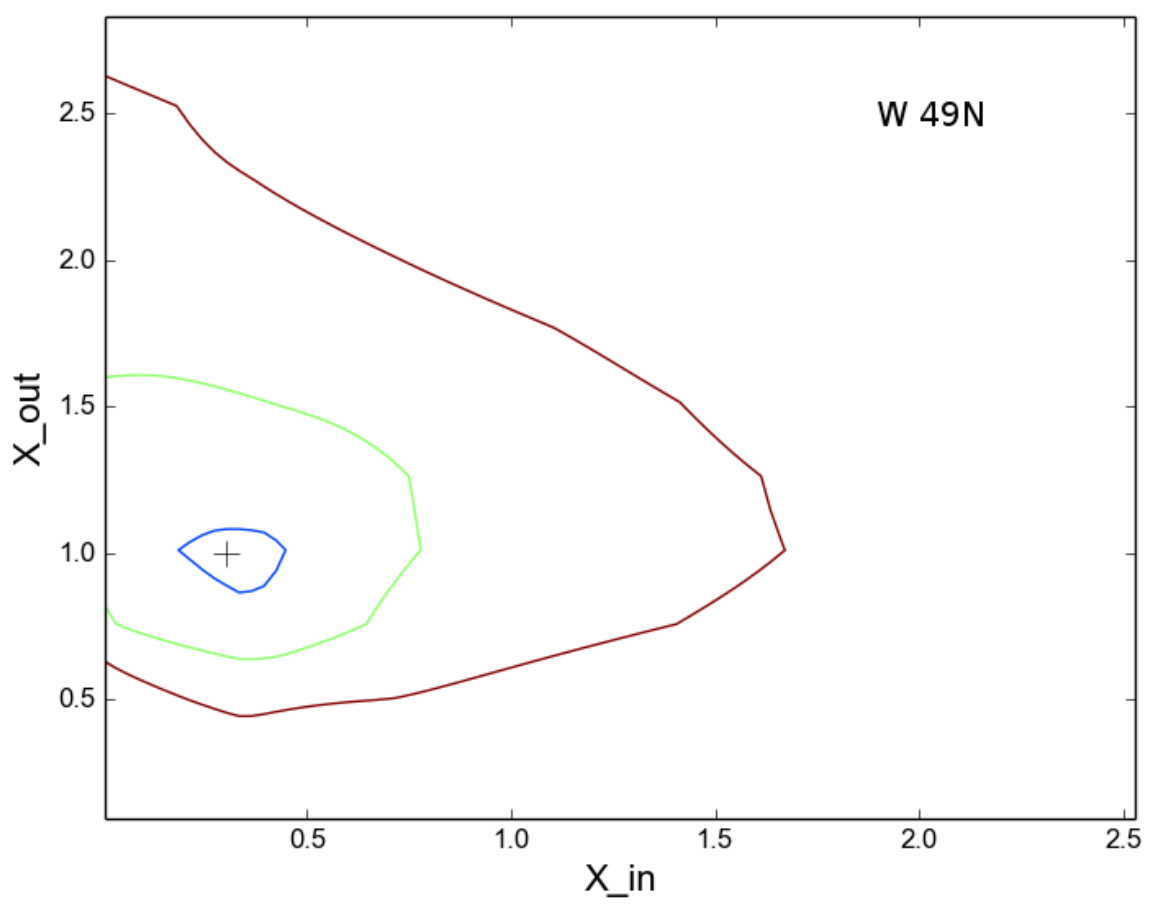

Figure 7. $X_{\text {in }}$ and $X_{\text {out }}$ HDO abundance contours at $1 \sigma, 2 \sigma, 3 \sigma$ for $\chi^{2}$. The best-fit model is represented by the symbol " $+\left(X_{\text {in }}=\mathrm{a} \times 10^{-8}, X_{\text {out }}=\mathrm{b} \times 10^{-10}\right)$.

ratios are consequently not well-constrained. We estimated that the inner $\mathrm{HDO} / \mathrm{H}_{2} \mathrm{O}$ ratio is about $(1-4) \times 10^{-4}$ within the range found in other high-mass star-forming regions (Jacq et al. 1990; Gensheimer et al. 1996, Emprechtinger et al. 2013; Liu et al. 2013). The $\mathrm{HDO} / \mathrm{H}_{2} \mathrm{O}$ ratio varies between the inner and outer regions of high-mass protostars. The water deuterium fractionation decreases from the cold outer regions to the warm inner regions. The same trend is also present in low-mass protostars (Coutens et al. 2013 and 2014). The difference could be explained by the gradient of deuteration within interstellar ices. Only the external ice layers evaporate in the cold envelope through non-thermal processes, whereas the inner part of ice mantles evaporates only in the hot core (Taquet et al. 2014). The $\mathrm{HDO} / \mathrm{H}_{2} \mathrm{O}$ ratio in the bulk of ice mantle preserves the past physical and chemical conditions which materials experienced, while the $\mathrm{HDO} / \mathrm{H}_{2} \mathrm{O}$ ratio in active surface layers reflects local physical and chemical conditions (Furuya et al. 2015). The enrichment of deuterium in water ice should mostly occur in the latter prestellar core and/or protostellar phases, where interstellar UV radiation is heavily attenuated and CO is frozen out. Another possibility for the decrease of water deuterium fractionation toward the inner regions would be the additional water vapor formation at high temperatures $(\mathrm{T}>200-300 \mathrm{~K})$ thorough reactions: $\mathrm{O}+\mathrm{H}_{2} \longrightarrow \mathrm{OH}+\mathrm{H}$ and $\mathrm{OH}+\mathrm{H}_{2} \longrightarrow \mathrm{H}_{2} \mathrm{O}+\mathrm{H}$, which would decrease the $\mathrm{HDO} / \mathrm{H}_{2} \mathrm{O}$ ratios. However, it requires that a large amount of oxygen is in atomic form rather than in molecules in the high density inner regions.

\section{SUMMARY}

Using CSO observations of HDO low-excitation transitions, as well as previous observations of HDO high excitations and $\mathrm{H}_{2}^{18} \mathrm{O}$ low-excitation transitions from the literature, we determined the inner and outer $\mathrm{HDO}$ abundances, as well as the $\mathrm{HDO} / \mathrm{H}_{2} \mathrm{O}$ outer ratios toward three high-mass star-forming 
Table 8. Best fit model parameters.

\begin{tabular}{llllclc}
\hline Source & $n_{0}\left(\mathrm{~cm}^{-3}\right)$ & $T_{0}(\mathrm{~K})$ & $X_{\text {in }}(\mathrm{HDO})$ & Range $(2 \sigma)$ & $X_{\text {out }}(H D O)$ & Range $(2 \sigma)$ \\
\hline G34.26+0.15 & $1.0 \times 10^{8}$ & 200 & $3.7 \times 10^{-8}$ & $2.7-5.6 \times 10^{-8}$ & $7.8 \times 10^{-11}$ & $0.2-2.1 \times 10^{-10}$ \\
W51e $1 / \mathrm{e}_{2}$ & $1.8 \times 10^{8}$ & 230 & $1.7 \times 10^{-8}$ & $1.1-2.4 \times 10^{-8}$ & $7.0 \times 10^{-11}$ & $0.3-1.1 \times 10^{-10}$ \\
W49N & $2.5 \times 10^{8}$ & 300 & $0.3 \times 10^{-8}$ & $0.2-0.5 \times 10^{-8 \star}$ & $1.0 \times 10^{-10}$ & $0.8-1.6 \times 10^{-10}$ \\
\hline
\end{tabular}

${ }^{\star}$ Range $(1 \sigma)$

regions: G34.26 + 0.15, W51e $1 / \mathrm{e}_{2}, \mathrm{~W} 49 \mathrm{~N}$. We derived HDO abundances of $X_{\text {in }}=(0.3-3.7) \times 10^{-8}$ (for $T \geq 100 \mathrm{~K}$ ) and $X_{\text {out }}=(7.0-10.0) \times 10^{-11}$ (for $T<100 \mathrm{~K}$ ), (see Table 8), and HDO $/ \mathrm{H}_{2} \mathrm{O}$ outer ratios of $(1.8-3.1) \times 10^{-3}$ (see Table 9). With this study, we showed that the $509 \mathrm{GHz}$ transition can provide good constraints on the HDO abundance in the transition region between the hot core and colder envelope, and that the $465 \mathrm{GHz} \mathrm{HDO}$ transition is a very good probe of the outer envelope of massive protostars. These transitions could help for more advanced modeling of water in highmass sources. The $\mathrm{HDO} / \mathrm{H}_{2} \mathrm{O}$ ratios were also found to be higher in the cold outer envelopes than in the hot cores, as already determined for two high mass sources. What is important the model is very simple, easy to implement, and not GPU-intensive, and provides a starting point for more sophisticated analysis.

The author thanks the referee very much for the highly constructive comments and suggestions. The author is grateful to N. Flagey for providing the reduced HIFI data of $\mathrm{H}_{2}^{18} \mathrm{O}$. M.K. would like to thank Maryvonne Gerin and Darek Lis for fruitful discussions and a careful reading of the manuscript. The work was carried out within the framework of the European Associated Laboratory "Astrophysics Poland-France" and also supported by the Science and High Education Ministry of Poland, grants N20339/3334. This research is based on observations from the Caltech Submillimeter Observatory, which is operated by the California Institute of Technology under cooperative agreement with the National Science Foundation (AST-0838261). 
Table 9. Comparison of HDO abundance between different sources

\begin{tabular}{|c|c|c|c|c|c|}
\hline Source & $X_{i n}(H D O)$ (best fit) & $X_{\text {out }}(H D O)$ (best fit) & $\left(\mathrm{HDO} / \mathrm{H}_{2} \mathrm{O}\right)_{i n}$ & $\left(\mathrm{HDO} / \mathrm{H}_{2} \mathrm{O}\right)_{\text {out }}$ & Ref. \\
\hline \multicolumn{6}{|c|}{ Low-mass protostars } \\
\hline L1448-mm & $\sim 4.0 \times 10^{-7}$ & $\leq 3.0 \times 10^{-9}$ & $\ldots$ & $\ldots$ & 1 \\
\hline \multirow[t]{3}{*}{ IRAS 16293-2422 } & $1.0 \times 10^{-7}$ & $1.5 \times 10^{-10}$ & $3 \times 10^{-2}$ & $\leq 2 \times 10^{-3}$ & 2 \\
\hline & $1.7 \times 10^{-7}$ & $8.0 \times 10^{-11}$ & $3.4 \times 10^{-2}$ & $5.0 \times 10^{-3}$ & 3 \\
\hline & $\ldots$ & $\ldots$ & $(9.2 \pm 2.6) \times 10^{-4}$ & $\ldots$ & 4 \\
\hline \multirow[t]{4}{*}{ NGC 1333-IRAS2A } & $8.0 \times 10^{-8}$ & $7.0 \times 10^{-10}$ & $\geq 1.0 \times 10^{-2}$ & $\sim 7 \times 10^{-2}$ & 5 \\
\hline & $\ldots$ & $\ldots$ & $(3.0-80) \times 10^{-3}$ & $\ldots$ & 6 \\
\hline & $\cdots$ & $\cdots$ & $1.0 \times 10^{-3}$ & $\cdots$ & 7 \\
\hline & $\ldots$ & $\ldots$ & $(7.4 \pm 2.1) \times 10^{-4}$ & $\ldots$ & 8 \\
\hline \multirow[t]{3}{*}{ NGC 1333-IRAS4B } & $\ldots$ & $\ldots$ & $\leq 6.0 \times 10^{-4}$ & $\ldots$ & 9 \\
\hline & $1.0 \times 10^{-8}$ & $1.2 \times 10^{-10}$ & $(1.0-37) \times 10^{-4}$ & $\cdots$ & 10 \\
\hline & $\ldots$ & $\ldots$ & $(5.9 \pm 1.7) \times 10^{-4}$ & $\ldots$ & 8 \\
\hline \multirow[t]{4}{*}{ NGC 1333-IRAS 4A-NW } & $\ldots$ & $\ldots$ & $\left(19.1 \pm 5.4 \times 10^{-4}\right.$ & $\ldots$ & 8 \\
\hline & $7.5 \times 10^{-9}$ & $1.2 \times 10^{-11}$ & $(4.0-30) \times 10^{-4}$ & $\cdots$ & 10 \\
\hline & $\ldots$ & $\ldots$ & $(5.0-30) \times 10^{-3}$ & $\ldots$ & 6 \\
\hline & Intel & nediate-mass protostar & & & \\
\hline \multirow[t]{2}{*}{ NGC 7192 FIRS2 } & $4.0 \times 10^{-8}$ & $\ldots$ & $\ldots$ & $\ldots$ & 11 \\
\hline & & h-mass hot cores * & & & \\
\hline \multirow[t]{5}{*}{ G34.26+0.15 } & $3.7 \times 10^{-8}$ & $7.8 \times 10^{-11}$ & $3.7 \times 10^{-4}$ & $3.1 \times 10^{-3}$ & 12 \\
\hline & $6.0 \times 10^{-8}$ & $5.0 \times 10^{-12}$ & $3.0 \times 10^{-4}$ & $(1.9-4.9) \times 10^{-4}$ & 13 \\
\hline & $2.0 \times 10^{-7}$ & $8.0 \times 10^{-11}$ & $(3.5-7.5) \times 10^{-4}$ & $(1.0-2.2) \times 10^{-3}$ & 14 \\
\hline & $4.6 \times 10^{-9}$ & $\ldots$ & $1.1 \times 10^{-4}$ & $\ldots$ & 15 \\
\hline & $\sim 2.7 \times 10^{-8}$ & $\ldots$ & $4.0 \times 10^{-4}$ & $\ldots$ & 16 \\
\hline $\mathrm{W} 51 \mathrm{e}_{1} / \mathrm{e}_{2}$ & $1.7 \times 10^{-8}$ & $7.0 \times 10^{-11}$ & $1.7 \times 10^{-4}$ & $2.8 \times 10^{-3}$ & 12 \\
\hline \multirow[t]{3}{*}{$\mathrm{W} 49 \mathrm{~N}$} & $3.0 \times 10^{-9}$ & $1.0 \times 10^{-10}$ & $3.0 \times 10^{-5}$ & $1.8 \times 10^{-3}$ & 12 \\
\hline & $1.5 \times 10^{-9}$ & $\ldots$ & $6.3 \times 10^{-5}$ & $\ldots$ & 15 \\
\hline & $\sim 2.2 \times 10^{-8}$ & $\ldots$ & $3.0 \times 10^{-4}$ & $\ldots$ & 16 \\
\hline W 33A & $2.0 \times 10^{-7}$ & $1.0 \times 10^{-8}$ & $\ldots$ & $\ldots$ & 17 \\
\hline NGC 6334 I & $1.3 \times 10^{-10}$ & $\ldots$ & $2.1 \times 10^{-4}$ & $\ldots$ & 18 \\
\hline $\mathrm{W} 3(\mathrm{OH}) /\left(\mathrm{H}_{2} \mathrm{O}\right)$ & $\ldots$ & $\ldots$ & $(2.0-6.0) \times 10^{-4}$ & $\ldots$ & 19 \\
\hline AFGL 2591 & $1.0 \times 10^{-7}$ & $2.0 \times 10^{-8}$ & $\ldots$ & $\ldots$ & 17 \\
\hline NGC 7538 IRS1 & $1.0 \times 10^{-7}$ & $4.0 \times 10^{-9}$ & $5.0 \times 10^{-4}$ & $(4.0-400.0) \times 10^{-3}$ & 17 \\
\hline \multirow[t]{2}{*}{ Sgr B2(M) } & $1.5 \times 10^{-9}$ & $1.3 \times 10^{-11}$ & $\ldots$ & $\ldots$ & 20 \\
\hline & $2.0 \times 10^{-9}$ & $\ldots$ & $1.8 \times 10^{-4}$ & $\ldots$ & 15 \\
\hline Orion KL & $\sim 4.5 \times 10^{-8}$ & $\ldots$ & $3.0 \times 10^{-3}$ & $\ldots$ & 21 \\
\hline
\end{tabular}

${ }^{\star}$ The fractional abundances of water in the hot core: $X_{i n}\left(H_{2} O\right) \sim 1.0 \times 10^{-4}$ (e.g. Boonman 2003, Snell et al. 2000, Herpin et al. 2012, Emprechtinger et al. 2013) are taken in the inner $\mathrm{HDO} / \mathrm{H}_{2} \mathrm{O}$ ratio estimation.

References - (1) Codella et al. 2010; (2) Parise et al. 2005; (3) Coutens et al. 2012; (4) Persson et al. 2014 on scale $\leq 300$ AU; (5) Liu et al. 2011; (6) Taquet et al. 2013; (7) Visser et al. 2013; (8) Persson et al. 2014,on scale $\leq 300$ AU; (9) Jørgensen et al. 2010, on scale $\leq 50$ AU; (10) Coutens et al. 2013; (11) Fuente et al. 2012; (12) This work; (13) Liu et al. 2013; (14) Coutens et al. 2014; (15) Gensheimer et al. 1996; (16) Jacq et al. 1990; (17) van der Tak et al. 2006; (18) Emprechtinger et al. 2013; (19) Helmich et al. 1996; (20) Comito et al. 2010; (21) Neill et al. 2013; (22) Lecuyer, C. et al. 1998. 


\section{REFERENCES}

Beuther, H., Schilke, P., \& Menten, K. M. 2002, ApJ, 566, 945

Boonman, A. M. 2003, in ESA-SP, Vol. 456, 67

Brown, P. D., \& Millar, T. J. 1989, MNRAS, 237, 661

Campbell, M. F., Garland, C. A., \& Deutsh, L. K. 2000, ApJ, 536, 816

Caselli, P., Vastel, C., Ceccarelli, C., \& et al. 2008, A\&A, 492, 703

Cazaux, S., Caselli, P., \& Spaans, M. 2011, ApJ, 741,34

Ceccarelli, C., Hollenbach, D. J., \& Tielens, A. G. G. M. 1996, ApJ, 471, 400

Chavarría, L., Herpin, F., Jacq, T., \& et al. 2010, A\&A, 521, 37

Choi, Y., van der Tak, F. F. S., van Dischoeck, E. F., \& et al. 2015, A\&A, 576, 85

Codella, C., Ceccarelli, C., Nisini, B., \& et al. 2010, A\&A, 522, 1

Coutens, A., Vastel, C., \& Hincelin, U. 2014, MNRAS, 445, 1299

Daniel, F., Dubernet, M. L., \& Grosjean, A. 2011, A\&A, 536, 76

De Pree, C. G., Wilner, D. J., \& Goss, W. M. 2000, A\&A, 540, 308

Draine, B. T. 2003, ARA\&A, 41, 241

Emprechtinger, M., Lis, D. C., Rollfs, R., \& et al. 2013, ApJ, 761, 61

Faure, A., Wiesenfeld, L., Scribano, Y., \& et al. 2012, MNRAS, 420, 699

Fish, V. L., Reid, M. J., Wilner, D. J., \& et al. 2003, ApJ, 587, 701

Flagey, N., Goldsmith, P Fand Lis, D. C., \& et al. 2013, ApJ, 762, 11

Fraser, H. J., Bisshop, S. E., Pontoppidan, K. M., \& et al. 2005, MNRAS, 356, 1283

Fraser, H. J., Collins, M. P., \& McCoustra, M. R. 2001, MNRAS, 327, 1165

Fuente, A., Caselli, P., Coey, C. M., \& et al. 2012, A\&A, 540, 75

Garay, G., \& Rodriguez, L. F. 1990, ApJ, 362, 191

Gensheimer, P. D., Mauersberger, R., \& Wilson, T. L. 1996, A\&A, 314, 281

Goldsmith, P. F., Bergin, E. A., \& Lis, D. C. 1997, ApJ, 491, 615

Gordon, K. D., Galliano, F., Hony, S., \& et al. 2010, A\&A, 518, 89

Gordon, M. A., \& Jewell, P. R. 1987, AJ, 323, 766
Gwinn, C. R., Moran, J. M., \& Reid, M. 1992, ApJ, 393, 149

Hajigholi, M., Persson, C. M., Wirstrom, E. S., \& et al. 2016, A\&A, 585, 158

Hatchell, J., \& van der Tak, F. F. S. 2003, A\&A, 409, 589

Helmich, F. P., van Dischoeck, E. F., \& Jansen, D. J. 1996, A\&A, 313, 589

Herpin, F., Chavarria, L., van der Tak, F. F. S., \& et al. 2012, A\&A, 542, 76

Hildebrand, R. H. 1983, QJRAS, 24, 267

Hill, M. A., Thompson, M. G., \& Burton, A. J. 2006, MNRAS, 368, 1223

Hunter, T. R., Neugebauer, G., \& Benford, D. J. 1998, ApJ, 493, 97

Jacq, T., Wamsley, C. M., Henkel, C., \& et al. 1990, A\&A, 228, 447

Keto, E. R., Ho, P. T. P., \& Reid, M. J. 1987, ApJ, 323, 117

Kulczak-Jastrzębska, M. 2016, AcA, 66, 239

Lampton, M., Margon, B., \& Bowyer, S. 1976, ApJ, 208, 177

Liu, F. C., Parise, B., Wyrowski, F., \& et al. 2013, A\&A, 550, A37

Lodders, K. 2003, ApJ, 591, 1220L

MacDonald, G. M., Gibb, A. G., Habing, R. J., \& Millar, T. J. 1996, A\&AS, 119, 333

Marseille, M. G., van der Tak, F. F. S., Herpin, F., \& Jacq, T. 2010, A\&A, 522, 74

Minier, V., Burton, M. G., Hill, T., \& et al. 2005, A\&A, 429, 945

Parise, B., Caux, E., Castets, A., \& et al. 2005, A\&A, 431, 547

Pety, J. 2005, in SF2A-2005: Semaine de l'Astrophysique Francaise, ed. T. Casoli, J. M. Contini, \& L. Pagani (Published by EdP-Sciences), 721

Pickett, H. M., Poytner, R. L., Cohen, E. A., \& et al. 1998, jqrst, 60, 883

Reid, M. J., \& Ho, P. T. P. 1985, ApJ, 288, L17

Roelfsema, P. R., Helmich, F. P., Yeyssier, D., \& et al. 2012, A\&A, 537, 17

Rolffs, R., Schilke, P., Comito, C., \& et al. 2010, A\&A, 521, 46

Rybicki, G. B., \& Hummer, D. G. 1991, A\&A, 245, 171

Sato, M., Reid, M. J., Brunthaler, A., \& Menten, K. M. 2010, ApJ, 720, 1055 
Schröier, F. L., van der Tak, F. F. S., \& van Dischoeck, E. F. 2005, A\&A, 432, 369

Schulz, A., Gusten, R., Walmsey, C. M., \& et al. 1991, A\&A, 246, 55

Shu, F. H. 1977, ApJ, 214, 488

Sievers, A. W., Mezger, P. G., \& Bordeon, M. A. 1991, A\&A, 251, 231

Snell, R. L., Howe, J. E., Ashby, M. L. N., \& et al. 2000, A\&A, 539, 97

Taquet, V., Charnley, S. B., \& Sipilä, O. 2014, ApJ, 791, 1

Thompson, M. A., Hatchell, J., Walsh, A. J., \& et al. 2006, A\&A, 453, 1003

Tielens, A. G. G. M. 1983, A\&A, 119, 177

van der Tak, F. F. S., Chavarria, L., Herpin, F., \& et al. 2013, A\&A, 554, 83

van der Tak, F. F. S., Marseille, M. G., Herpin, F., \& et al. 2010, A\&A, 518, 107 van der Tak, F. F. S., van Dishoeck, E. F., Evans, N. J., \& et al. 2000, ApJ, 537, 283

van der Tak, F. F. S., Walmsley, C. M., Herpin, F., \& et al. 2006, A\&A, 447, 1101

van Dishoeck, E. F., Herbst, E., \& Neufeld, D. A. 2013, ChRv, 113, 9043

Viti, S., \& Williams, D. A. 1999, MNRAS, 305, 755

Ward-Thompson, D., \& Robson, E. I. 1990, MNRAS, 244, 458

Wiesenfeld, L., Scribano, Y., \& Faure, A. 2011, PCCP, 13, 8230

Wright, M., Goeran, S., \& Wilner, D. J. 1992, ApJ, 393, 225

Zhang, Q., \& Ho, P. T. P. 1997, ApJ, 488, 241

Zmuidzinas, J., Blake, A., Carlstrom, J., \& et. al. 1995, ApJ, 447, L125 